\title{
Digital Vessel Thrombosis After Early Cessation of Heparin Therapy Following Reimplantation: Two Case Reports
}

Kevin McGarry, MD; Michael Larsen, MD²; Mitchell A. Pet, MD, FACS²; Amy M. Moore, MD, FACS ${ }^{*}$

1 Department of Plastic and Maxillofacial Surgery, Ulster Hospital Dundonald, Northern Ireland

${ }^{2}$ Division of Plastic and Reconstructive Surgery, Department of Surgery, Washington University School of Medicine,

Saint Louis, Missouri, USA

${ }^{3}$ Department of Plastic and Reconstructive Surgery, The Ohio State University Wexner Medical Center, Columbus, Ohio, USA

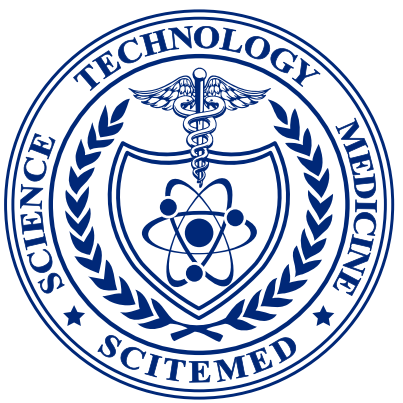

\begin{abstract}
Although a recognized phenomenon in cardiology, the implications of rebound heparin hypercoagulability following cessation of therapy in microsurgery is unreported. In this article we report two cases of late digital circulatory compromise following replantation, shortly after withdrawal of heparin. We propose potential consideration for changes in perioperative anticoagulation practice to reduce this risk. In conclusion, if heparin is instituted as the treatment of choice following microvascular repair, continuation of heparin therapy until neovascularization has occurred may reduce the risk of the rebound hypercoagulation. Addition of aspirin or other antiplatelet agents as used in the acute coronary setting may also have a role at minimizing the effect.
\end{abstract}

\section{INTRODUCTION}

Rebound hypercoagulation following cessation of heparin therapy has previously been reported in the cardiology setting [1-3]. Initially it was recognized following reinfarction clustering within hours of discontinuation of unfractionated heparin infusion (UFI) during treatment of ST elevation myocardial infarction [4]. Reactivation of the coagulation system and elevated thrombin production following heparin withdrawal was proposed as the likely cause [3]. With loss of therapeutic heparin levels, prothrombotic factors interact with the original thrombus rapidly resulting in propagation, platelet recruitment and new ischaemia [5]. In vivo studies have further explained the molecular pathogenesis [2]. Increases in plasma prothrombin fragment 1+2 $(\mathrm{F} 1+2)$ and thrombin-antithrombin (TAT) levels were recorded on heparin withdrawal that were beyond those measured prior to commencing heparin therapy [1].

Although a recognized phenomenon in cardiology, the implications of rebound heparin hypercoagulability in microsurgery are as yet unreported. In both the elective and trauma microsurgical setting, heparin is a component of the postoperative protocol for many European and American micro-surgeons, despite limited evidence for its efficacy [6,7]. Propagation of thrombosis in a microvascular anastomosis is similar to the pathological progression of thrombosis propagation in coronary arteries, and can result in critical tissue loss.

In this article we report two cases of late digit circulatory compromise shortly after withdrawal of heparin therapy. Additionally, we propose potential consideration for changes in perioperative anticoagulation practice to reduce this risk.

\section{CASE REPORTS}

\section{Case 1}

A three-year-old right hand dominant healthy male who sustained near-complete amputation of his right index finger through the distal interphalangeal joint when both hands were caught in the hinge of metal portable truck ramp. Vaccines including tetanus were up to date and he had no current medical issues.

The patient was taken emergently to the operating theatre within 4 hours of injury, for revascularization of the digit. Open reduction and percutaneous pinning with volar plate, collateral ligament, flexor digitorum profundus repair and extensor dermodesis was carried out. The ulnar digital artery (UDA), nerve (UDN) and a vein were repaired primarily under the microscope without requirement of a vein graft. The patient tolerated the procedure well, with no flow issues on the operating table and was transferred to the Pediatric Intensive Care Unit (PICU) for standard flap / replant monitoring.

A heparin (500 units) infusion and $60.75 \mathrm{mg}$ of daily aspirin were commenced postoperatively. The room was kept warm, and a forced air warming blanket kept on the right hand at all times. On postoperative day three,

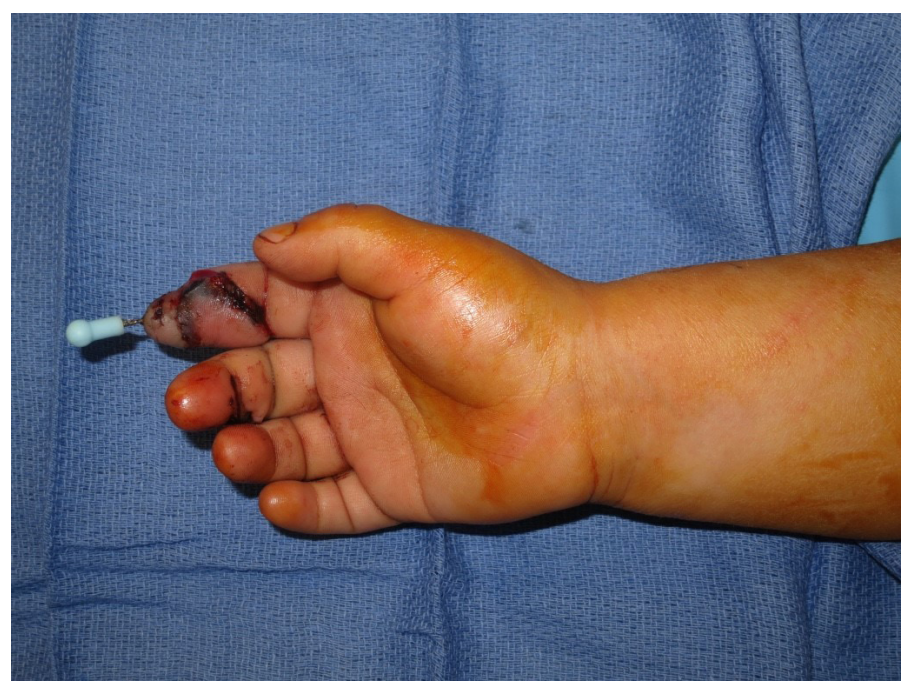

Figure 1. New onset fingertip ischemia following heparin withdrawal in Case 1. 


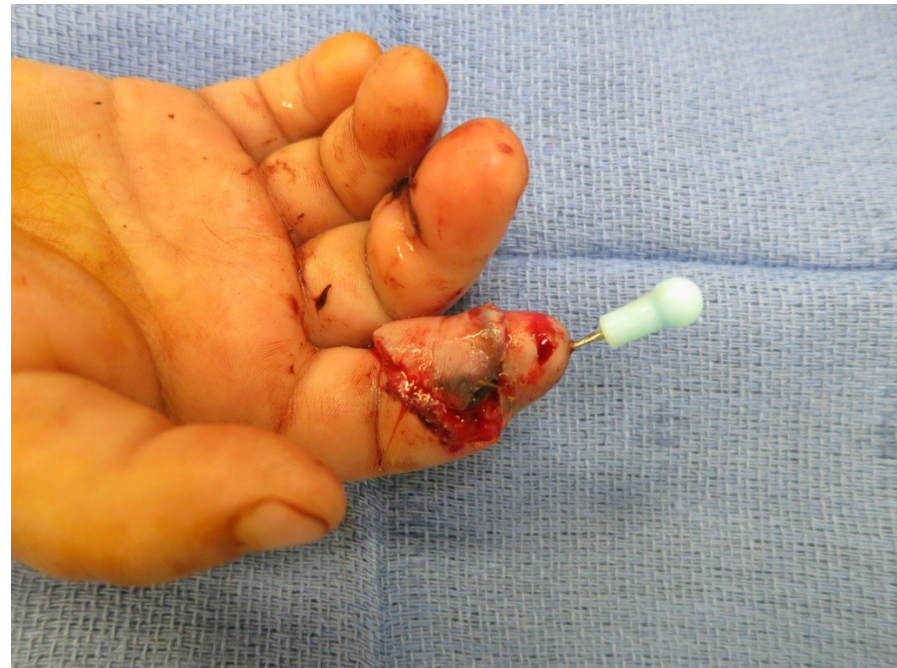

Figure 2. Image of fingertip following salvage in Case 1.

the heparin infusion was discontinued. He remained in the PICU with ongoing neurovascular checks. No other surgical or medical interventions that may have impacted upon circulation were performed in this time period (i.e., removal of warming blanket or change in other systemic factors). Approximately 21 hours later, the finger was noted to be cool and pale (Figure 1). No doppler signal was found. The patient returned to theatre emergently for exploration and an arterial thrombus at the anastomosis was noted. Flow was re-established, he was returned to the PICU postoperatively for ongoing neurovascular checks and recommenced on the heparin infusion (Figure 2).

This continued for a further five days post return to theatre at which stage he was transitioned to therapeutic enoxaparin for the remainder of his inpatient stay, for a total of seven days of postoperative anticoagulation. He was discharged on a further 30-day course of aspirin. At first outpatient follow-up the replanted digit was found to be viable with no further episodes of vascular compromise noted.

\section{Case 2}

A twenty-seven-year-old right hand dominant male presented with right thumb amputation by miter saw, through the proximal phalanx with sixhour cold ischemia time. No significant past medical history. Social history included smoker of one pack / day and current occupation as a carpenter.

He was taken to the operating room for emergent replantation, which included debridement, shortening and K-wire bony fixation. The flexor pollicis longus (FPL) and extensor pollicis longus (EPL) tendon were repaired. The radial digital artery (RDA) underwent microsurgical repair in addition to two veins and the UDN. Flow was restored without any intraoperative issue. The patient was bolused with 3000 units of heparin IV and placed on a 500-unit heparin infusion. A therapeutic 300mg aspirin suppository was given on transfer to the post-anesthesia care unit, then $81 \mathrm{mg}$ aspirin was commenced daily. Recovery was uneventful until day three post operatively when the heparin infusion turned off. Again, no other interventions in this time period that may have impacted upon circulation were carried out. Within two hours of ending the infusion the thumb became clinically congested requiring reinstitution of intravenous heparin, and therapeutic leaching. Inflow was maintained and the patient improved with the above measures therefore operative intervention was not required. The patient was subsequently discharged on daily aspirin and therapeutic enoxaparin for two weeks. At first outpatient follow-up the thumb was viable with no further episodes of vascular compromise.

\section{DISCUSSION}

The consequence of intravascular thrombosis was first described in the mid1800s by the German physician Rudolf Virchow [8]. It is only over the past three decades, however, that advances in immunohistochemistry and flow cytology have allowed molecular mapping of the processes underlying Virchow's original triad of hypercoagulability, vessel damage and blood flow stasis [9]. The cases we present of late thrombosis following the cessation of heparin infusion to make hand surgeons aware of this possible preventable sequela.

Those undergoing microsurgical reconstruction or digital revascularization have their thrombotic risk compounded by a range of injury and patient specific factors. Transection and trauma to the vessel wall leading to exposure of subendothelial structures to blood flow [10], which has been shown to have the greatest single impact on acute anastomotic thrombosis [11]. This acute insult is followed by postoperative inflammatory response, upregulation of endothelial P-selectin surface expression and relative vessel wall hypoxia during neo-endothelialization [12], which can take a further two six days in animal models to occur [13]. Localization of the tissue injury (e.g., extremities, high energy trauma or concurrent contamination or infection) further increase this risk [14].

Additional patient specific risks, namely dehydration and long periods of immobility, also exacerbate the likelihood of postoperative thrombosis [15]. In this setting a further pro-thrombotic insult, i.e., the acute withdrawal of heparin and subsequent thrombotic rebound, could be enough to produce microvascular thrombosis, as in the cases discussed. Interestingly this is in fact opposite to the more commonly recognized HITT (Heparin Induced Thrombocytopenia Thrombosis) phenomenon more commonly described in the hospitalized population. HITT occurs on initiation of heparin therapy rather than cessation.

Although no specific guidance exists in the microvascular setting to reduce the risk of rebound heparin thrombosis at this present time, a significant amount of cardiology guidelines have been produced addressing this event in acute coronary syndrome following heparin withdrawal. Aspirin has been shown to blunt the effects of rebound thrombosis after heparin withdrawal improving survival in cardiac patients [16]. The patients presented remained on aspirin during heparin withdrawal however, possibly highlighting the different pathogenesis in the microsurgical clinical scenario. This complicates drawing any direct conclusion over use of one or both drugs in this different clinical setting. If heparin is instituted however, we would recommend the continuation of therapy until vessel neovascularization has occurred (for a period of at least 6 days or longer based on animal studies [13]). Given the additional risk of side effects, i.e., bleeding or gastric ulceration, the decision to institute additional medications in the microvascular setting should be judicious and patient specific.

Rebound hypercoagulation following withdrawal of heparin therapy is a rare but recognized treatment sequelae. Given the period of inflammation that immediately follows microvascular repair in both the elective and trauma setting we would suggest maximizing patient factors through hydration and early mobility (i.e., walking, not digit/extremity mobility) as the primary treatment modality.

The use of heparin therapy in the post-operative period after revascularization or replantation remains controversial based on current evidence $[17,18]$. To date there is no high-level evidence supporting use of any medications in microsurgery as a means to reduce risk of arterial or venous thrombosis. Higher level studies are urgently needed to investigate the clinical efficacy of antithrombotic medications in microsurgery; however, given the small failure rates in modern practice, these will need to be large multicenter trials in order to reach sufficient power [6].

If heparin is instituted in the perioperative setting, the continuation of heparin therapy until neovascularization has occurred (for a period of at least 6 days or longer [13]) may reduce the risk of the rebound hypercoagulation. Addition of aspirin or other antiplatelet agents as used in the acute 
coronary setting may have a role at minimizing this effect [19]. However, as in the first case presented, Aspirin alone was not effective in preventing circulatory compromise. As no current international guidelines exist, use of any anticoagulation therapy should be carefully considered. Additionally, a full explanation of the potential side effects of anticoagulation prior to institution should be given to patients.

\section{CONCLUSION}

If heparin is instituted as the treatment of choice following microvascular repair, continuation of heparin therapy until neovascularization has occurred may reduce the risk of the rebound hypercoagulation. Addition of aspirin or other antiplatelet agents as used in the acute coronary setting may also have a role at minimizing the effect.

\section{ARTICLE INFORMATION}

*Correspondence: Amy M. Moore, MD, FACS. Professor and Chair, Department of Plastic and Reconstructive Surgery, The Ohio State University Wexner Medical Center, 915 Olentangy River Rd \# 2140, Columbus, Ohio, USA. Email: amy.m.moore@ osumc.edu

Received: Nov. 12, 2020; Accepted: May 10, 2021; Published:Jun. 25, 2021

DOI: 10.24983/scitemed.imj.2021.00141

Ethics Approval and Consent to Participate: The study is in accordance with the ethical standards of the 1964 Helsinki declaration and its later amendments or comparable ethical standards. The University of Washington does not require ethical approval for reporting individual cases or case series. Written informed consent was obtained from the patient(s) for their anonymized information to be published in this article.

Funding: The study did not receive any specific grant from funding agencies in the public, commercial, or not-for-profit sectors.

Conflict of Interest: The authors report no financial or other conflict of interest relevant to this article, which is the intellectual property of the authors.

Copyright $\odot 2021$ The Author(s). This is an open-access article distributed under the terms of the Creative Commons Attribution 4.0 International License (CC-BY).

\section{REFERENCES}

1. Bijsterveld NR, Moons AH, Meijers JC, et al. Rebound thrombin generation after heparin therapy in unstable angina. A randomized comparison between unfractionated and low-molecular-weight heparin. J Am Coll Cardiol 2002;39(5):811-817.

2. Becker RC, Spencer FA, Li Y, et al. Thrombin generation after the abrupt cessation of intravenous unfractionated heparin among patients with acute coronary syndromes: Potential mechanisms for heightened prothrombotic potential. J Am Coll Cardiol 1999;34(4):1020-1027.

3. Linder R, Oldgren J, Egberg $\mathrm{N}$, et al. The effect of a low molecular mass thrombin inhibitor, inogatran, and heparin on thrombin generation and fibrin turnover in patients with unstable coronary artery disease. Eur Heart J 1999;20(7):506-518.

4. Granger CB, Hirsch J, Califf RM, et al. Activated partial thromboplastin time and outcome after thrombolytic therapy for acute myocardial infarction: Results from the GUSTO-I trial. Circulation 1996;93(5):870-878.

5. Theroux P, Waters D, Lam J, Juneau M, McCans J. Reactivation of unstable angina after the discontinuation of heparin. N Engl J Med 1992;327(3):141-145.

6. Kearns MC, Baker J, Myers S, Ghanem A. Towards standardization of training and practice of reconstructive microsurgery: An evidence-based recommendation for anastomosis thrombosis prophylaxis. Eur J Plast Surg 2018;41(4):379-386.

7. Jokuszies A, Herold C, Niederbichler AD, Vogt PM. Anticoagulative strategies in reconstructive surgery--clinical significance and applicability. Ger Med Sci 2012;10:Doc01.

8. Bagot CN, Arya R. Virchow and his triad: A question of attribution. Br J Haematol 2008;143(2):180-190.

9. Brass LF, Diamond SL. Transport physics and biorheology in the setting of hemostasis and thrombosis. J Thromb Haemost 2016;14(5):906-917.

10. Khouri RK, Cooley BC, Kenna DM, Edstrom LE. Thrombosis of microvascular anastomoses in traumatized vessels: Fibrin versus platelets. Plast Reconstr Surg 1990;86(1):110-117.

11. Acland R. Thrombus formation in microvascular surgery: An experimental study of the effects of surgical trauma. Surgery 1973;73(5):766-771.

12. Poredos $P$, Jezovnik MK. The role of inflammation in venous thromboembolism and the link between arterial and venous thrombosis. Int Angio/ 2007;26(4):306311.

13. Walker LN, Ramsay MM, Bowyer DE. Endothelial healing following defined injury to rabbit aorta. Depth of injury and mode of repair. Atherosclerosis 1983;47(2):123130.

14. Khouri RK, Cooley BC, Kunselman AR, et al. A prospective study of microvascular free-flap surgery and outcome. Plast Reconstr Surg 1998;102(3):711-721.

15. Engbers MJ, Blom JW, Cushman M, Rosendaal FR, van Hylckama Vlieg A. The contribution of immobility risk factors to the incidence of venous thrombosis in an older population. J Thromb Haemost 2014;12(3):290-296.

16. Amsterdam EA, Wenger NK, Brindis RG, et al. 2014 AHA/ACC guideline for the management of patients with non-ST-elevation acute coronary syndromes: A report of the American College of Cardiology/American Heart Association Task Force on Practice Guidelines. Circulation 2014;130(25):e344-426.

17. Chen CM, Ashjian P, Disa JJ, Cordeiro PG, Pusic AL, Mehrara BJ. Is the use of intraoperative heparin safe? Plast Reconstr Surg 2008;121(3):49e-53e.

18. Kaciulyte J, Losco L, Maruccia M, et al. Postsurgical antithrombotic therapy in microsurgery: Our protocol and literature review. Eur Rev Med Pharmacol Sci 2019;23(10):4448-4457.

19. Gionis MN, Ioannou CV, Kontopodis N, Balalis K, Elalamy I, Gerotziafas GT. Heparin resistance and coagulation activation rebound effect after anticoagulant withdrawal: Beneficiary effect of adjuvant antiplatelet therapy. Int Angiol 2016;35(2):170-177. 